\title{
The effect of reward magnitude differences on choosing disadvantageous decks in the Iowa Gambling Task
}

\author{
Ruud van den Bos*, Bart B. Houx, Berry M. Spruijt \\ Ethology and Welfare, Faculty of Veterinary Medicine, Utrecht University, Yalelaan 2, \\ NL-3584 CM Utrecht, The Netherlands
}

Received 7 November 2004; accepted 5 May 2005

Available online 10 August 2005

\begin{abstract}
In the Iowa Gambling Task, participants have to develop a long-term profitable monetary scenario in a situation of uncertainty and a conflict between the chance of encountering an immediate large reward (US\$ 100) in two long-term loosing decks (A and B; US\$ - 250 per 10 cards) and the chance of encountering an immediate small reward (US\$ 50) in two long-term winning decks (C and D; US $\$+250$ per 10 cards). The ratio of the immediate rewards in decks $A / B$ and $C / D$ is thus $2: 1$. Here, we manipulated these differences in reward magnitude between the advantageous $(\mathrm{C} / \mathrm{D})$ and disadvantageous $(\mathrm{A} / \mathrm{B})$ decks, while keeping the net gains and losses per 10 cards the same, to assess the impact of the conflict between immediate and distant pay-off on choice behaviour. Participants selected less cards from disadvantageous decks and won more money when the reward magnitude difference was decreased, $A / B: C / D=1: 1$, while they selected more cards from disadvantageous decks and lost more money when reward magnitude differences were increased, $A / B: C / D=4: 1$ and 6:1. This study shows that the outcome in the Iowa Gambling Task is sensitive to differences between the magnitude of immediate rewards in the advantageous and disadvantageous decks.

(C) 2005 Elsevier B.V. All rights reserved.
\end{abstract}

Keywords: Iowa Gambling Task; Planned behaviour; Reward magnitude; Dopamine

\section{Introduction}

In the Iowa Gambling Task (IGT), participants have to develop a long-term profitable monetary scenario in a situation of uncertainty, and a conflict between the chance of encountering an immediate large reward (US\$ 100) in two long-term loosing decks (A and B; US\$ -250 per 10 cards) and the chance of encountering an immediate small reward (US\$ 50) in two long-term winning decks (C and D; US $\$ 250$ per 10 cards; Bechara et al., 1994). Thus far, the IGT has been mainly used as research tool in humans to study how decision-making is guided by somatic markers, i.e. somatosensory marker signals that bias decisions through their association with emotions and feelings (see Bechara et al., 2000a; Tranel et al., 2000 for extensive reviews). As knowledge on neurobiological mechanisms of decision-making has increased considerably recently, the

\footnotetext{
* Corresponding author. Tel.: +31 30253 4373; fax: +31 302539227

E-mail address: R.vandenBos@las.vet.uu.nl (R.van den Bos).
}

IGT may be used as a tool to study decision-making in more detail. Here, we study the effect of differences in reward magnitude between the advantageous $(\mathrm{C} / \mathrm{D})$ and disadvantageous $(\mathrm{A} / \mathrm{B})$ decks.

Thus far, studies have shown that the amygdala and ventromedial prefrontal cortex are important structures in developing a choice for long-term winning decks C and D (Bechara et al., 1997, 1998, 1999). Furthermore, it has been shown that the dopaminergic system is important in the early stages of the test (Bechara et al., 2001). This dopaminergic activity may be related to exploring the different decks and responding to rewards (Fiorillo et al., 2003). The ventral striatum may be critically involved herein (Knutson et al., 2001). A serotonergic component appears to be present in the later stages of the test (Bechara et al., 2001). This serotonergic activity may be related to regulating the extent to which individuals continue or maintain their choice behaviour for the best long-term option, as opposed to be tempted to visit the long-term loosing decks that contain immediate large rewards, i.e. self-control, as serotonin has 
been implicated in controlling levels of impulsive behaviour (Higley et al., 1996a, 1996b; Mehlman et al., 1994, 1995) or controlling the extent to which mesolimbic dopaminergic activity gains control over behaviour (Katz, 1999). The dorsolateral prefrontal cortex may be critically involved herein (Ernst et al., 2002; McClure et al., 2004; Ridderinkhof et al., 2004).

The differential involvement of these neurotransmitter systems suggests a transition from one set of neuronal structures to another as the test progresses, i.e. from the reward system, necessary for learning the best long-term option by assessing and integrating trial-by-trial wins and losses, to a cognitive control system, aiding in maintaining to choose once chosen options for which pay-off lies ahead in the future (Bechara and Damasio, 2002; Ernst et al., 2002; McClure et al., 2004; Ridderinkhof et al., 2004; Tranel et al., 2000; Shizgal and Arvanitogiannis, 2003). McClure et al. (2004) have demonstrated that the activity of this cognitive control system (delta system in their nomenclature) is stronger than the activity of the reward system (beta system) when decisions are made for options where pay-off lies ahead in future, while the beta system has a slightly higher activity than the delta system when decisions are made for which pay-off is immediate. The IGT contains a conflict between immediate and long-term pay-off. It has been shown that individual differences exist in the extent to which subjects are able to withhold responding to the immediate pay-offs of the disadvantageous decks (Bechara and Damasio, 2002; van den Bos, 2004; Crone et al., 2004). The IGT is therefore suited to measure the extent to which individuals are able to maintain successful long-term behaviour in the face of temptations that trigger the reward system. We therefore decided to study the effect of reward magnitude differences between decks $\mathrm{A} / \mathrm{B}$ and $\mathrm{C} / \mathrm{D}$ in the Iowa Gambling Task on choosing cards from the disadvantageous decks.

We increased the immediate rewards in decks A and B to $€ 200$ (Euros rather than US Dollars were used in the present experiment) or $€ 300$ while leaving the overall losses per 10 cards in decks A and B the same. The latter means that the overall structure of the task is the same across the test conditions, and rationally seen the outcomes should be nearly the same. However, as the reward system is increasingly more active, when differences between immediate pay-offs of the different decks increase (Fiorillo et al., 2003; Knutson et al., 2001) weakening thus the impact of the cognitive control system (McClure et al., 2004), we predicted that participants choose more from disadvantageous decks as differences between immediate pay-off become larger. As we wished to assess the impact of the conflict of immediate pay-off between decks A/B and $\mathrm{C} / \mathrm{D}$ as such we decreased the immediate rewards in decks $\mathrm{A}$ and $\mathrm{B}$ to $€ 50$ while leaving the overall losses the same. We predicted that participants choose less of disadvantageous decks, as no differences exist between immediate pay-off.

\section{Materials and methods}

\subsection{Participants}

Forty-seven subjects ( $n=25$ males and $n=22$ females; mean age: 28.7 years, S.D. $=10.9$ years) were assigned to the different experimental groups such that groups were balanced for age and gender composition. The final group compositions were: 50:50 condition: 10:8 (females:males), $31.1 \pm 2.7$ (S.E.M.) years; 100:50 condition: 4:5, $27.2 \pm 3.0$ years; 200:50 condition: 5:7, $27.1 \pm 3.5$ years; $300: 50$ condition: $2: 4,26.3 \pm 5.0$ years. No significant differences existed for gender $($ Chi-square $=1.138$, d.f. $=3, p>0.05)$ and age $(F(3$, $39)=0.501, p>0.05$ ) between groups. Participants were in general from academic and/or middle-class background.

\subsection{Procedure}

The experiment was run on a PC. The original Iowa Gambling Task was programmed on a computer using Microsoft Excel with VBA macros. This computer version allowed us to change the differences between the immediate reward magnitudes and overall losses or gains per block of 10 cards in an easy and straightforward way.

The subject was seated in front of the computer screen and after reading test instructions, filling in name, gender and age, the test appeared. It consisted of four 'decks' of cards on which the subject could click using the mouse. Below each deck, it was graphically and numerically indicated how many cards were still present in the deck. Whenever no cards were left in the deck and subjects would still click on the 'deck', a message in red appeared that the deck was empty, and this choice would not add up to the number of trials. Above the decks, a card was shown on which the information of the chosen card appeared. The information on this card was the same as on the original cards (Bechara et al., 1994). In addition, the net score was shown on this card. At the bottom of the screen, a counter indicated how much money subjects had. At the right side of the screen, this was also graphically represented by a purple bar that moved up and down a numeric scale in line with the amount of money won or lost. When subjects had made 100 choices, a message in red appeared stating that the game was over. We added to the instructions of the original test (Bechara et al., 1994) that the computer did not manipulate the amount of money of the cards from the different decks after choices were made, i.e. cards in decks were independent of one another, as pilot studies showed that people tended to think that the computer manipulated the amount of money on the cards on basis of their choices (see also Bechara et al., 2000b).

In the original task, decks A and B contain cards, which always have written ' +100 ' (Euro) on them indicating the gain. In deck A, five cards per block of 10 cards are present which contain additional figures ranging from ' -150 ' to ' -350 ', indicating the amount that needs to be subtracted 
from the gain. In deck B, five cards are present per block of 10 cards, which contain either a ' 0 ' $(n=4)$ or a ' -1250 ' $(n=1)$. Decks A and B lead to an overall loss of $€ 250$ per block of 10 cards. Decks C and D contain cards, which always have written ' +50 ' on them indicating the gain. In deck $\mathrm{C}$, five cards per block of 10 cards are present which contain additional figures ranging from ' -25 ' to ' -75 ', indicating the amount that needs to be subtracted from the gain. In deck D, five cards are present per block of 10 cards, which contain either a ' 0 ' $(n=4)$ or a ' -250 ' $(n=1)$. Decks $\mathrm{C}$ and $\mathrm{D}$ lead to an overall $€ 250$ gain per block of 10 cards.

We used four different conditions for immediate reward magnitude differences between decks $\mathrm{A} / \mathrm{B}$ and C/D: 50:50 (1:1), 100:50 (2:1), 200:50 (4:1) and 300:50 (6:1). The overall losses and gains per block of 10 cards were kept the same, i.e. $€-250$ per block of 10 cards in decks A and B, and $€+250$ per block of 10 cards in decks $\mathrm{C}$ and $\mathrm{D}$. The losses per card were automatically adjusted to achieve the latter. In all conditions, the order of cards in the decks was the same (cf. original task of Bechara et al., 1994).

\subsection{Dependent variables}

The dependent variables were: the difference of the number of cards between the advantageous decks (C/D) and disadvantageous decks $(\mathrm{A} / \mathrm{B})$ per block of 20 choices, a standard measure to assess task learning; the number of cards from decks A and B after 100 choices to assess whether differences in performance between test conditions are due to changes in all or only one of the disadvantageous decks; the cumulative amount of money gained or lost per block of 20 choices, as the task requires participants to earn as much money as possible, and monetary budget reflects adequately whether they succeed in this or not.

\subsection{Statistics}

One-way and two-way analyses of variance (ANOVA) were used whenever appropriate. Factors are indicated in Section 3. Greenhouse-Geisser corrected degrees of freedom and $p$-values were used whenever Mauchly's test of sphericity was significant, i.e. when the sphericity assumption was violated. Planned comparisons between different test conditions were run after significant ANOVAs using Helmert contrasts. All analyses were run in SPSS v9.0 for PC. Differences were considered to be significant when $p \leq 0.05$. A trend was considered when $0.05<p \leq 0.10$. Results were considered not significant when $p>0.10$ (NS). All statistics are two-tailed unless otherwise indicated.

\section{Results}

Two individuals (one male and one female) in the 300:50 condition were discarded from the analysis as their performance differed strongly from the remainder of the group: 19 and 24 cards from the disadvantageous decks compared to 46-66 (range) for the remainder of the group. In fact, these two individuals scored the lowest and fourth lowest number of cards from the disadvantageous decks in the entire dataset.

Fig. 1, panel A, shows that subjects in the 50:50, 100:50 and 200:50 groups improved their performance over trial blocks, and that subjects in the 50:50 group chose more cards from the advantageous decks than subjects in the 100:50, 200:50 and 300:50 groups, especially in the early trial blocks (two-way ANOVA [reward magnitude, trial block (repeated measure)]: trial block: $F(3.2 ; 131.6$ [Greenhouse-Geisser corrected] $)=5.161, \quad p \leq 0.002$; reward magnitude $\times$ trial block: $F(9.6 ; 131.6$ [Greenhouse-Geisser corrected] $)=0.926$, NS; reward magnitude: $F(3,41)=8.159, p \leq 0.001)$. Planned comparisons using Helmert contrasts showed that the 50:50 condition differed significantly from the 100:50 condition $(p \leq 0.044)$, and that the 200:50 and 300:50 conditions combined differed from the 100:50 condition ( $p \leq 0.044)$, as well as differed from one another $(p \leq 0.038)$.

With increasing reward magnitude the number of cards from both deck A and deck B increased (Fig. 1, panel B; two-way ANOVA [reward magnitude, deck A/B (repeated)]: reward magnitude: $F(3,41)=8.076, p \leq 0.001$; reward magnitude $\times$ deck A/B: $F(3,41)=0.463$, NS $)$. Independent of reward magnitude differences deck $B$ was preferred over deck A (deck A/B: $F(1,41)=45.246, p \leq 0.001)$.

The 50:50 group won money throughout, whereas the remainder of the groups lost money such that the amount of money lost increased, as reward magnitude differences increased, with the 300:50 group showing a deep trough half-way the task (Fig. 1, panel C; two-way ANOVA [reward magnitude, trial block (repeated measure)]: reward magnitude $\times$ trial block: $\quad F(8.6 ; 118.1 \quad$ [Greenhouse-Geisser corrected] $)=9.866, p \leq 0.001$; reward magnitude: $F(3$, $41)=14.434, \quad p \leq 0.001)$. Planned comparisons using Helmert contrasts showed that the 50:50 condition differed significantly from the 100:50 condition $(p \leq 0.005)$, and that the 200:50 and 300:50 conditions combined differed significantly from the 100:50 condition ( $p \leq 0.012)$, but did not differ from one another.

\section{Discussion}

The present study showed that increasing the differences in reward magnitude between decks $\mathrm{A} / \mathrm{B}$ and $\mathrm{C} / \mathrm{D}$ from $100: 50$ (2:1), i.e. the difference in the original task (Bechara et al., 1994), to 200:50 (4:1) and 300:50 (6:1) increased the number of cards taken from the disadvantageous decks and increased the amount of money lost, while decreasing the differences in reward magnitude between decks $\mathrm{A} / \mathrm{B}$ and $\mathrm{C} /$ D from 100:50 to 50:50 (1:1) decreased the number of cards taken from the disadvantageous decks and decreased the amount of money lost. 
This experiment revealed that whenever differences between decks are introduced in terms of immediate rewards (100:50, 200:50 and 300:50) subjects choose more cards from the disadvantageous decks than when no such differences are present. In the 50:50 condition punishments exclusively determine differences between decks, and no conflict exists between the decks that may tempt the individual to explore the test environment any further or

\section{Number of CD-AB cards}

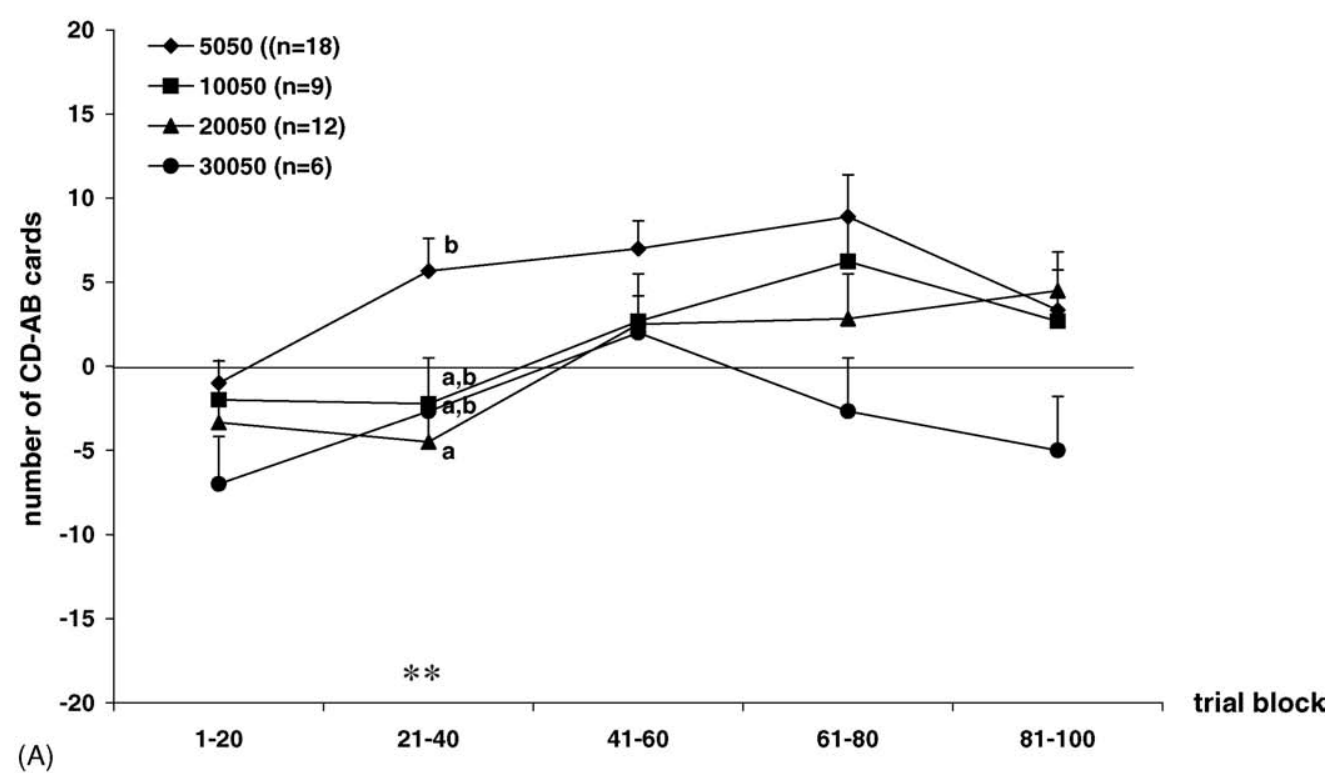

Number of cards from deck $A$ and $B$ after 100 trials

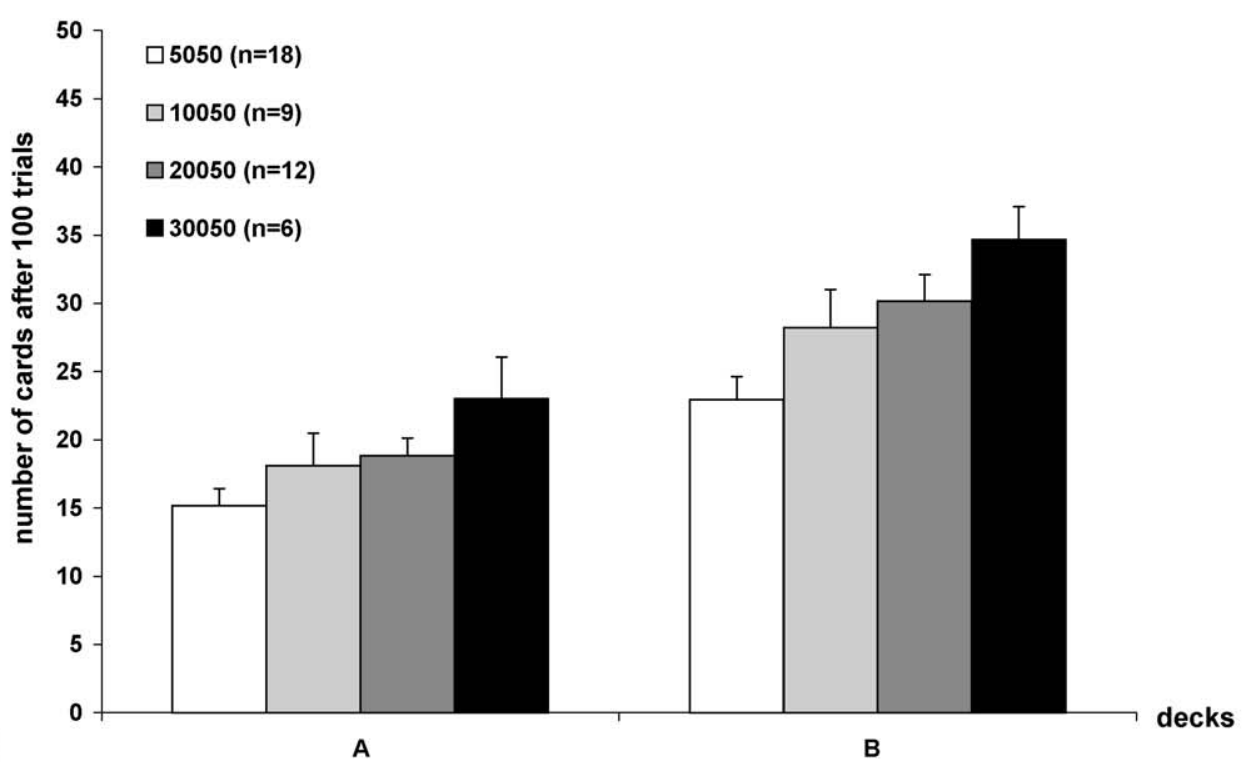

Fig. 1. (Panel A) Test performance across blocks of 20 choices expressed as the number of cards from decks $C$ and $D(C D)$ minus the number of cards from decks $\mathrm{A}$ and $\mathrm{B}(\mathrm{AB})$ per block of 20 choices for the different reward magnitude groups (mean + S.E.M.). Values $>0$ indicate an improvement of performance, i.e. more cards from the advantageous decks than disadvantageous decks. Asterisks indicate a significant effect after one-way ANOVA $\left({ }^{* *} p \leq 0.01\right)$; groups with the same small letters are not different from one another (Student-Newman-Keuls test following significant one-way ANOVA). A trend ( $p \leq 0.07)$ was found for trial block 61-80; a post hoc SNK revealed that subjects in the 50:50 group differed from subjects in the 300:50 group. (Panel B) The mean number (+S.E.M.) of cards chosen from decks A and B after 100 choices for the different reward magnitude groups. For statistics, see text. (Panel C) Mean cumulative amount of money (+S.E.M.) per block of 20 of choices for the different reward magnitude groups. Asterisks indicate a significant effect after one-way ANOVA $\left({ }^{*} p \leq 0.05\right.$, ${ }^{* *} p \leq 0.01$ and ${ }^{* * *} p \leq 0.001$ ); groups with the same small letters are not different from one another (Student-Newman-Keuls test following significant one-way ANOVA). 


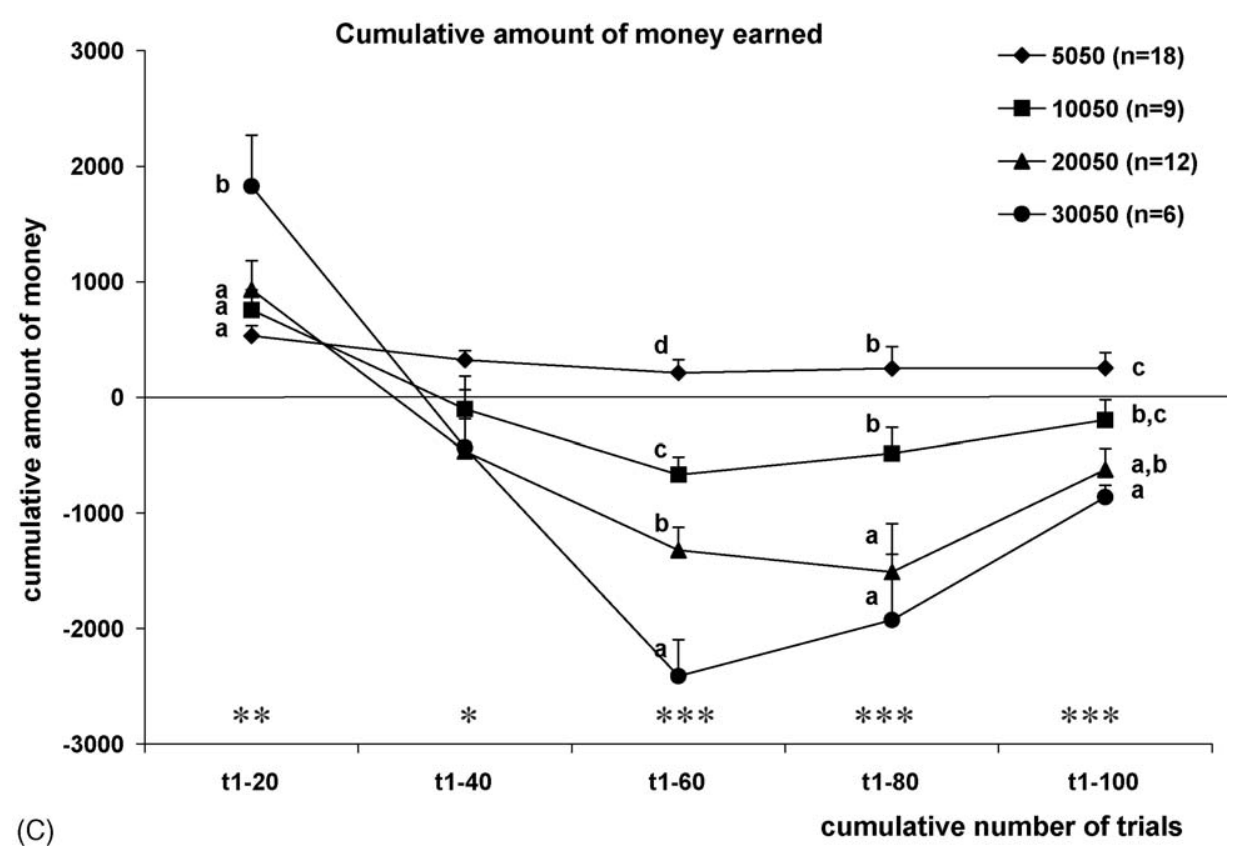

Fig. 1. (Continued).

respond to larger rewards. The subjects in the 50:50 group changed their behaviour to taking cards from the advantageous decks $\mathrm{C} / \mathrm{D}$, before subjects changed their behaviour in any of the other groups, i.e. from trial 20 onwards rather than from trial 40 onwards.

In contrast, the data show that the larger the reward differences between decks the more the individual is tempted to choose from the disadvantageous decks even when the odds in terms of the net amount of money are clearly against him or her. Deck B was clearly preferred over deck A (cf. Crone and Van der Molen, 2004; Crone et al., 2004), which may be due to the fact that deck B contains more, and more regularly, cards with immediate wins than deck A (see Bechara et al., 1994). The increase of choices from the disadvantageous decks was not due to an increase in choice behaviour for either deck alone. This shows that individuals visit the disadvantageous decks overall more.

It should be noted that choosing cards from the disadvantageous decks in the 300:50 condition in the second half of the test rapidly decreased losses of participants, although the final amount of money was still less than in the other test conditions. In other words, this behaviour may be reinforced by the rapid increase of the amount of money. This emphasises a more general phenomenon of this task under conditions of differences in reward magnitude. When losses have been incurred, subjects are faced with the dilemma that one card from deck $\mathrm{A}$ or $\mathrm{B}$ in terms of the maximum amount of money is equivalent to two $(€ 100)$, four $(€ 200)$ or six $(€ 300)$ cards from deck $\mathrm{C}$ or $\mathrm{D}$ in terms of the maximum amount. Thus, in terms of making up rapidly for losses of money, choosing cards from deck A or B seems a better option than choosing cards from deck $\mathrm{C}$ or $\mathrm{D}$, because of the chance of rapid success. Because of the order of the cards in the decks (see Bechara et al., 1994), unknown of course to subjects, choosing cards from deck A or B after strong losses, indeed may pay-off for a number of choices. However, given the strong losses that subjects already have incurred by choosing cards from deck A or B, it is clear that this behaviour is risky as subjects do not know the consequences of their subsequent choices for cards of these decks. Therefore, it could be that subjects in the 300:50 condition actually gave up on the test because of the strong negative budget, kept on choosing cards from decks $\mathrm{A}$ and $\mathrm{B}$, with an incidental increase of their budget.

The data overall suggest that the reward magnitude difference in the IGT is a powerful tool to manipulate the extent to which individuals are able to suppress the temptation to keep responding to the large rewards in the disadvantageous decks. Recently, it was shown that the (mesolimbic) dopamine system is more strongly activated under conditions of uncertainty when differences between trial-by-trial rewards are large than when they are small (Fiorillo et al., 2003). This activity occurs prior to obtaining the reward and has been suggested to reinforce risk-taking behaviour or the willingness to invest in rewards, i.e. to help the individual to engage in behaviour ('risk-taking'/ 'attention') that promotes learning about relevant environmental events (Fiorillo et al., 2003; Schultz, 2004; Shizgal and Arvanitogiannis, 2003; Spruijt et al., 2001). The ventral striatum may be critically involved herein (van den Bos et al., 2002; Knutson et al., 2001; Matthews et al., 2004; Spruijt et al., 2001). This activity has been explicitly associated with (excessive) gambling (Fiorillo et al., 2003; Shizgal and Arvanitogiannis, 2003). In the original Iowa Gambling Task, subjects encounter reward magnitude 
differences between the long-term disadvantageous decks A/ $\mathrm{B}$ on one hand, and the long-term advantageous decks C/D on the other (Bechara et al., 1994) as they learn the task features. Thus, as they visit the different decks, dopaminergic activity may be strongly activated due to reward magnitude differences. As individuals learn the relevant features of the decks and have decided their strategy for the remainder of the task, a shift may occur from systems dependent on dopamine that allow to learn about the relevant items of the game, including the ventral striatum, to systems dependent on serotonin that allow to continue the chosen strategy, including the dorsolateral prefrontal cortex (van den Bos, 2004; Ernst et al., 2002; McClure et al., 2004; Ridderinkhof et al., 2004). Thus, decreasing reward magnitude differences from 100:50 to 50:50 may lead to a weaker dopaminergic activity, while increasing these differences to a more strongly activated dopaminergic activity which could act to reinforce visits to decks A and B (cf. Fiorillo et al., 2003). The latter may lead to a disbalance between the serotonin system and dopamine system in favour of the dopamine system and consequently many visits to decks A and B (Katz, 1999).

It has been shown that the incentive value of rewards depends upon previous experiences with these rewards, which may also explain the differences between the groups. Rats show a higher running speed for 16 pellets when they are used to 1 pellet rather than 16 throughout (positive contrast) and a lower running speed for 16 pellets when they were used to 256 pellets rather than 16 pellets throughout (negative contrast; Lieberman, 1993, pp. 228-231). In the present study, such a contrast effect may occur as subjects explore the environment and switch between decks: an increase in the subjective value of the large reward (positive contrast) and a decrease in the subjective value of the small reward (negative contrast). This increases the temptation to visit the decks with the large rewards, and this temptation may increase as reward magnitude differences increase. The increase in ventral striatal dopaminergic activity may then be related to an increased exploratory activity induced or amplified by this contrast effect.

Thus, next to the amygdala and ventromedial prefrontal cortex (Bechara et al., 2000a; Tranel et al., 2000) the ventral striatum may be involved in the IGT as well (cf. Knutson et al., 2001). While physiological measures, such as skin conductance responses, may be used to study the differences between the role of the amygdala and ventromedial prefrontal cortex (Bechara et al., 1999), reward magnitude differences may be potentially used to study the sensitivity to ventral striatal dopaminergic activity. It is tempting to speculate that the two subjects that we discarded from the 300:50 condition and who were extremely insensitive to these test conditions may have a lower sensitivity of ventral striatal dopaminergic activity. It should be noted that including these subjects in the analyses would not have changed the overall picture (data not shown).
In conclusion, manipulating reward magnitude differences in the Iowa Gambling Task leads to either decreased risk behaviour, when there are no reward magnitude differences, or increased risk behaviour with increasing reward magnitude differences.

\section{Acknowledgements}

The authors would like to thank Sietse Jonkman and students from the Neuroscience and Cognition Master Programme from Utrecht University for their help in collecting the data. Furthermore, they would like to thank the referees and Dr. Ridderinkhof for constructive comments that improved the quality of the manuscript.

\section{References}

Bechara, A., Damasio, A.R., Damasio, H., Anderson, S.W., 1994. Insensitivity to future consequences following damage to human prefrontal cortex. Cognition 50, 7-15.

Bechara, A., Damasio, H., Tranel, D., Damasio, A.R., 1997. Deciding advantageously before knowing the advantageous strategy. Science 275, 1293-1295.

Bechara, A., Damasio, H., Tranel, D., Anderson, S.W., 1998. Dissociation of working memory from decision making within the human prefrontal cortex. The Journal of Neuroscience 18, 428-437.

Bechara, A., Damasio, H., Damasio, A.R., Lee, G.P., 1999. Different contributions of the human amygdala and ventromedial prefrontal cortex to decision-making. The Journal of Neuroscience 19, 54735481.

Bechara, A., Damasio, H., Damasio, A.R., 2000a. Emotion, decision making and the orbitofrontal cortex. Cerebral Cortex 10, 295-307.

Bechara, A., Tranel, D., Damasio, H., 2000b. Characterization of the decision-making deficit of patients with ventromedial prefrontal cortex lesions. Brain 123, 2189-2202.

Bechara, A., Damasio, H., Damasio, A.R., 2001. Manipulation of dopamine and serotonin causes different effects on covert and overt decision making, Society of Neuroscience Abstracts 465.5.

Bechara, A., Damasio, H., 2002. Decision-making and addiction (part I): impaired activation of somatic states in substance dependent individuals when pondering decisions with negative future consequences. Neuropsychologia 40, 1675-1689.

van den Bos, R., Houx, B.B., Spruijt, B.M., 2002. Cognition and emotion in concert in human and nonhuman animals. In: Bekoff, M., Allen, C., Burghardt, G. (Eds.), The Cognitive Animal; Empirical and Theoretical Perspectives on Animal Cognition. The MIT Press, Cambridge (MA), pp. 97-103.

van den Bos, R., 2004. Emotion and cognition. In: Bekoff, M. (Ed.), .The Handbook of Animal Behavior. Greenwood Press, Westport (CT), pp. 554-557.

Crone, E.A., van der Molen, M.W., 2004. Developmental changes in real life decision making: performance on a gambling task previously shown to depend on the ventromedial prefrontal cortex. Developmental Neuropsychology 25 (3), 251-279.

Crone, E.A., Somsen, R.J.M., Beek, van B., van der Molen, M.W., 2004. Heart rate and skin conductance analysis of antecedents and consequences of decision making. Psychophysiology 41, 531-540.

Ernst, M., Bolla, K., Mouratidis, M., Contoreggi, C., Matochik, J.A., Kurian, V., Cadet, J.-L., Kimes, A.S., London, E.D., 2002. Decisionmaking in a risk-taking task: a PET study. Neuropsychopharmacology 26, 682-691. 
Fiorillo, C.D., Tobler, P.N., Schultz, W., 2003. Discrete coding of reward probability and uncertainty by dopamine neurons. Science $299,1898-$ 1902.

Higley, J.D., Mehlman, P.T., Poland, R.E., Taub, D.M., Vickers, J., Suomi, S.J., Linnoila, M., 1996a. CSF testosterone and 5-HIAA correlate with different types of aggressive behaviors. Biological Psychiatry 40, 10671082.

Higley, J.D., Mehlman, P.T., Higley, S.B., Fernald, B., Vickers, J., Lindell, S.O., Taub, D.M., Suomi, S.J., Linnoila, M., 1996b. Excessive mortality in young free-ranging male nonhuman primates with low cerebrospinal fluid 5-hydroxyindoleacetic acid concentrations. Archives of General Psychiatry 53, 537-543.

Katz, L.D., 1999. Dopamine and serotonin: integrating current affective engagement with longer-term goals. Behavioral and Brain Sciences 22, 527.

Knutson, B., Adams, C.M., Fong, G.W., Hommer, D., 2001. Anticipation of increasing monetary reward selectively recruits nucleus accumbens. The Journal of Neuroscience 21 (RC159), 1-5.

Lieberman, D.A., 1993. Learning; Behavior and Cognition, second ed. Brooks/Cole Publishing Company, Pacific Grove, California.

Matthews, S.C., Simmons, A.N., Lane, S.D., Paulus, M.P., 2004. Selective activation of the nucleus accumbens during risk-taking decision making. NeuroReport 15, 2123-2127.

McClure, S.M., Laibson, D.I., Loewenstein, G., Cohen, J.D., 2004. Separate neural systems value immediate and delayed monetary rewards. Science 306, 503-507.
Mehlman, P.T., Higley, J.D., Faucher, I., Lilly, A.A., Taub, D.M., Vickers, J., Suomi, S.J., Linnoila, M., 1994. Low CSF 5-HIAA concentrations and severe aggression and impaired impulse control in nonhuman primates. American Journal of Psychiatry 151, 1485-1491.

Mehlman, P.T., Higley, J.D., Faucher, I., Lilly, A.A., Taub, D.M., Vickers, J., Suomi, S.J., Linnoila, M., 1995. Correlation of CSF 5-HIAA concentration with sociality and timing of emigration in free-ranging primates. American Journal of Psychiatry 152, 907-913.

Ridderinkhof, K.R., van den Wildenberg, W.P.M., Segalowitz, S.J., Carter, C.S., 2004. Neurocognitive mechanisms of cognitive control: the role of prefrontal cortex in action selection, response inhibition, performance monitoring, and reward-based learning. Brain and Cognition 56, 129 140.

Schultz, W., 2004. Neural coding of basic reward terms of animal learning theory, game theory, microeconomics and behavioural ecology. Current Opinion in Neurobiology 14, 139-147.

Shizgal, P., Arvanitogiannis, A., 2003. Gambling on dopamine. Science 299, 1856-1858.

Spruijt, B.M., van den Bos, R., Pijlman, F.T.A., 2001. A concept of welfare based on reward evaluating mechanisms in the brain: anticipatory behaviour as an indicator for the state of reward systems. Applied Animal Behaviour Science 72, 145-171.

Tranel, D., Bechara, A., Damasio, A.R, 2000. Decision making and the somatic marker hypothesis. In: Gazzaniga, M.S. (Ed.), .The New Cognitive Neurosciences. A Bradford Book, Cambridge (MA), pp. 1047-1061. 